\section{Plug and play electric micromotor boost available}

Having an electric micromotor on the dental chair can greatly enhance efficiency, power and tactile sense, according to B.A. International. This is particularly relevant when doing restorative dentistry and working with zirconia and metal. The combination of electric micromotor and speed increasing handpiece allows for turbine-like speeds while maintaining the torque at all speeds.

Up to now, to convert dental chairs - often only equipped with air motors - to electric would have mostly meant retrofitting costly built-in kits.

A perfect solution that B.A. can offer is an ingenious 'plug \& play' micromotor which allows every dental unit to be equipped with an electric micromotor in under two minutes at a price far less than a built-in kit.

The B.A. EM420 brushless motor with LED and internal spray gives a speed range from 1,000-40,000 rpm and allows the clinician to program their most used speeds as well.

In combination with the top of the range titanium B.A. 1:5 speed increasing handpiece, complete with DLC (diamond-like carbon) coated bearings and three years warranty, one would be hard pushed to find a more cost-effective solution in this important area.

For more information, contact B.A. on www.bainternational. co.uk or call 01604777700 .

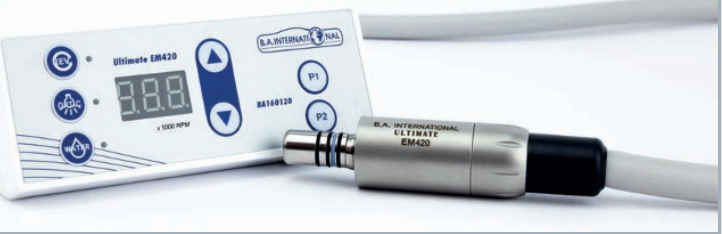

Key to success

The key to success is to make clinical procedures simpler. This is why Ketac Universal glass ionomer restorative from 3M Oral Care has only four steps: to make your treatments easier and your successes greater.

- Step one - the clinician activates the capsule

- Step two - the clinician mixes the capsule

- Step three - the clinician places Ketac Universal restorative in bulk

- Step four - the clinician finishes the restoration.

In addition to this simplified procedure, Ketac Universal glass ionomer restorative does not need conditioner, coating or layering, meaning it helps cut down on chair time too.

To get the key to success, contact the $3 \mathrm{M}$ Oral Care team today.

For more information, call 0800626578 or visit www.3M. co.uk/Dental.

\section{Trust in adhesive excellence}

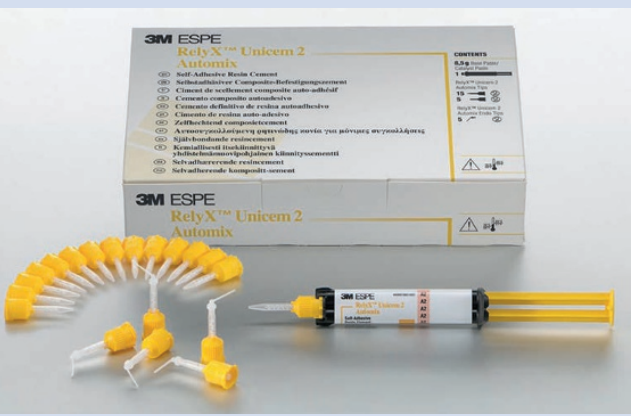

3M Oral Care has always been dedicated to developing solutions that benefit dental practitioners every day. RelyX Unicem selfadhesive resin cement is one such solution as one of the world's most clinically proven self-adhesive resin cements.

It was awarded the Dental Advisor's 'Best self-adhesive cement' in 2017, praised for its low debond rate, low sensitivity, easy excess removal and very reliable outcomes.

It has also been shown to decrease the number of working steps for a reduced risk of error.

To put your trust in a product proven to deliver results time after time, discover RelyX Unicem self-adhesive resin cement from 3M Oral Care.

For more information, call 0800626578 or visit www.3M. co.uk/Dental.

\section{Lighting the way for preventive dentistry}

Often, the most effective approach to dental care is to prevent a problem before it becomes an issue, but to do this, dental professionals need equipment that allows them to make early, accurate assessments.

Enter the CALCIVIS imaging system. By using a unique recombinant photoprotein that binds with the free calcium ions that are indicative of active demineralisation, this new technology provides dental professionals with an unmistakable bioluminescent (light emitting) signal that can be used to identify dental caries at its earliest stages.

Following this signal, dental professionals can plan a reliable preventive treatment plan for

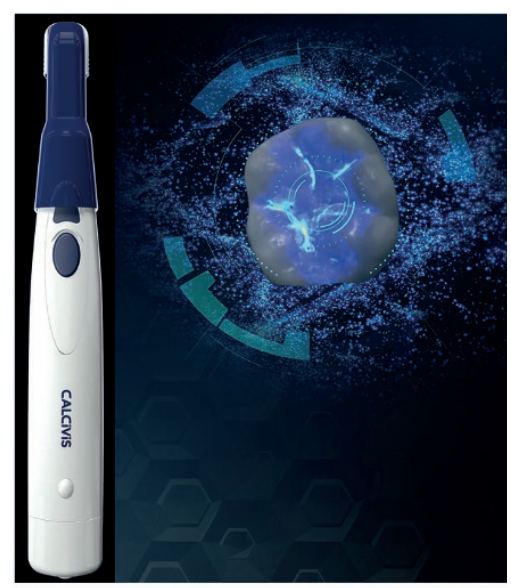

their patients, based on objective evidence. This then paves the way for remineralisation treatments that can reverse the effects of earlystage tooth decay before they are irreparable.

Claiming to be the only product on the market that can accurately and reliably detect active demineralisation on the surface of individual teeth, the CALCIVIS imaging system has the potential to change the way dental professionals approach preventive dental care.

More information is available at www.CALCIVIS.com or by calling 01316585152 